\title{
Reduction of Crack Density in Ammonothermal Bulk GaN Growth
}

\author{
Edward Letts, Daryl Key and Tadao Hashimoto \\ SixPoint Materials, Inc., Buellton, CA 93427, USA, ed@spmaterials.com
}

\section{Introduction}

GaN devices have become commonplace for lighting applications, already

available in every hardware store in the U.S. This is despite GaN device's limitations caused by growing the epitaxial layer on foreign substrates or on "quasi-bulk" substrates produced by hydride vapor phase epitaxy (HVPE) [1,2]. However "true bulk" GaN substrates could further improve device performance in high power lighting applications by allowing higher current densities and reducing droop [3]. In addition, high quality $\mathrm{GaN}$ substrates could allow new GaN device structures to be achieved such as high power and high frequency devices. These high power high frequency devices have the potential to be much more efficient than current silicon based devices due to GaN's physical properties. The primary benefits of using $\mathrm{GaN}$ devices include reduced energy costs, higher power densities which allow for smaller volume devices, and higher switching frequencies which allows for reduced capacitance and inductance which can result in a large reduction in weight and volume. It is estimated that $25 \%$ of the worldwide annual energy consumption could be saved by wide spread adoption of highly efficient power electronics [4].

Impressive results have demonstrated the feasibility of using free standing $\mathrm{GaN}$ to achieve near ideal electronic performance [5]. To address the lack of "true bulk" free standing GaN, several technologies are being investigated with some success including bulk HVPE [6], Sodium flux method [7-9], both acidic and basic ammonothermal growth [10-13], and regrowth by HVPE on "true bulk" seeds [14]. However, none of these techniques have yet been able to produce a sufficient amount of wafers at low cost for industry adoption.

In this paper, we review our progress in producing wafers by the ammonothermal method at SixPoint Materials. In particular, a detailed X-ray diffraction study of seed and growth improved the selection of appropriate seeds and resulted in reduced crack formation. In addition, our growth was modified to a Near Equilibrium Ammonothermal 
(NEAT) method which combined with proper seed selection, has lead to crack free growth.

\section{Experimental Details}

Ammonothermal growths were carried out in autoclaves made of a $\mathrm{Ni}-\mathrm{Cr}$ based superalloy with a 1" inner diameter. The growth was modified to a condition we define as the NEAT method. The interior of the autoclave was divided into a nutrient region and a seed region with the use of a flow-restricting baffle(s). The growth temperature was in the range of $450-600^{\circ} \mathrm{C}$ and had an ammonia fill factor between $40-60 \%$. Unlike many other growth methods, the growth could occur on both the gallium and nitrogen surfaces. The resulting ammonia pressure was in the range of $100-300 \mathrm{MPa}$. Polycrystalline GaN and/or metallic Ga were used as nutrient. Free-standing GaN wafers produced by HVPE were used as seed crystals. A mineralizer was added to increase the solubility of $\mathrm{GaN}$ allowing better $\mathrm{GaN}$ transport and faster $\mathrm{GaN}$ growth.

Six commercial (002) oriented two inch HVPE seeds produced by different companies were obtained. Samples were cleaved into smaller pieces, typically $10 \mathrm{~mm} x$ $12 \mathrm{~mm}$, to allow multiple growth experiments to be done. The thicknesses of seeds and grown crystals were measured with a micrometer. Surface morphology was observed with a Nomarski microscope. Pictures were taken using a Canon Powershot SX510 HS. Structural quality was evaluated with full width half maximum (FWHM) of the X-ray rocking curve (XRC) from both (002) and (201) reflections. XRC was collected using a PANalytical high-resolution four-axis X-ray diffractometer with a $\mathrm{Cu}$ anode operated at $40 \mathrm{~mA}$ and $40 \mathrm{keV}$ and a four-crystal Ge (002) monochromator with either a full open beam or a reduced beam size of $0.3 \mathrm{~mm} \times 0.3 \mathrm{~mm}$. No slits were used at the detector.

Grids of different seeds' and growths' XRC FWHM values were obtained on the nitrogen and/or gallium surface with reduced beam size of $0.3 \mathrm{~mm}$ x $0.3 \mathrm{~mm}$ using a 9-24 hr long program. In our ammonothermal growths the nitrogen surface has the largest growth window and is the most repeatable. Because of the increased stability, all reported scans in this paper are on the nitrogen surface. Full optimization alignment scans were only taken in the very center of the sample. Taking 0.5 or $2 \mathrm{~mm}$ steps, lines of measurements were done forming a grid and/or line(s) across the wafer as received, 
after any processing, and after growth. The samples were always mounted in the same orientation with the program primarily stepping along the m direction. FWHM values were calculated using an algorithm as follows: A rectangular smoothing was performed with a smoothing ratio of $1 / 5$, then a parabola was fit to data points with an intensity between 30 and $70 \%$ of the maximum. A user viewed and corrected the algorithm whenever a multi-peak or other irregularity was detected.

Impurities in the grown crystals were quantified with secondary ion mass spectroscopy (SIMS), which was measured in Evans Analytical Group, LLC. Dislocation densities were found by dislocation counting Nomarski images after a 3 minute $\mathrm{KOH}$ $\mathrm{NaOH}$ molten etch. Dislocation densities values have been confirmed with other techniques such as cathodoluminescence.

Different treatments were investigated on each seed. Examples include preferentially etching surface damage from the nitrogen surface with hot $\mathrm{H}_{3} \mathrm{PO}_{4}$ acid, polishing, and annealing at elevated temperatures in $\mathrm{NH}_{3}$ environments.

Crystals were sliced into $\sim 550 \mu \mathrm{m}$ thick wafers in a commercially obtained multi wire saw. All wafer images shown were taken from the nitrogen side of the growth for consistency.

\section{Results and Discussion}

Crystals produced at SixPoint Materials by the ammonothermal method typically have had high local structural quality as measured by X-ray diffraction though with high oxygen impurity concentrations, typically low $10^{19} \mathrm{~cm}^{-3}$. Historically, SixPoint has been able to grow less than $1 \mathrm{~mm}$ crack free, however as the growth continues the curvature flips and cracks begin to appear. In an attempt to understand the effect of the underlying seed structure and solve the cracking problem, six different manufacturer's seeds were acquired.

Each seed was measured, cleaved into approximately twelve $10 \mathrm{~mm}$ x12 mm pieces, separated, and placed into different ammonothermal growths runs. Each growth run had approximately 6 different seeds allowing direct comparisons to be made. Each growth run conditions had nominally identical conditions. 
Grid measurement using a reduced beam spot of $0.3 \mathrm{~mm} \times 0.3 \mathrm{~mm}$ of the as received seed's nitrogen surface provided the mean XRD FWHM values shown in Table 1. Clear differences exist between the different manufacturer's seeds. FWHM values for the (002) reflection ranged from 99 to 582 arcsec and the (201) reflection had a range between 59 and 534 arcsecs. Also of interest was the large differences in standard deviations of the FWHM values, 6 to 55 arcsecs and 9 to 173 arcsecs for the (002) and (201) reflections respectively. Hence some manufacturers had a fairly uniform structural quality, while others were more inconsistent. For instance, Manufacturer 4 had a high mean 002 FWHM value of 180 arcsec but the lowest standard deviation of 6 arcsec while Manufacturer 5 had a better mean FWHM but a higher standard deviation.

Some of the high outlier values are partially due to an inconsistent Nitrogen surface finish by different manufacturers. Manufacturer 1 for instance had a relatively matte nitrogen surface and values measured on the Ga surface were better. The best results on Manufacturer 1's seeds were obtained by removing some of the surface damage with either an etch or a better polish. This example identifies one problem with ammonothermal growth being able to grow on both the nitrogen and gallium surfaces. Since substrate manufacturers traditionally focus on making the gallium surface epi-ready, the nitrogen surface may need additional processing to be appropriate for ammonothermal growth.

Other differences in grain structure were also observed such as large differences in the (002) FWHM and (201) FWHM ratios, with a range between 0.60 and 2.1. The difference in ratios was likely caused by different buffer layers or templates that may have been used. Plotted grid profiles of the six different manufacturer's as received seeds nitrogen surface (002) and (201) XRC FWHM values are summarized in Table 1 and shown in Figure 1.

Multiple seeds were placed into each autoclave run to allow for direct comparisons. Growth runs were $\sim 90$ days and resulted in a final thickness of 2.5-6 mm. Grown crystals had the same XRD characterization performed as the seed in the same orientation as the respective seeds. Typical growth for almost all seeds showed local improvement in FWHM values and a curvature flip as described above. However the crack density varied widely, which could degrade the X-ray FWHM value calculated by 
our algorithm near the crack if two distinct peaks were not observed. Distinct duel peaks can emerge in the XRD scans near a crack, with an angular separation up to 0.4 degrees. Additionally, growth on some seeds showed consistent peak splitting without any visible cracks, we believe this is caused by grains that propagate into the growth but become misaligned.

We suspected that the differences in crack densities between the different manufactured seeds were not caused by impurities. Since the crystals were cut into similar shapes and grown in either the same run or in nominally identical conditions, impurity concentrations profiles across the crystals are expected to be reasonably consistent between the ammonothermally grown crystals. However each HVPE seed is expected to have some differences in impurity levels since they were made by different manufacturers. It is expected that any differences in impurity concentrations between HVPE manufacturers is slight compared to the difference between impurities obtained in HVPE growth versus ammonothermal growth.

After characterization, the crystals were sliced in a multi-wire saw and recharacterized. The difference in crack density in obtained unpolished wafers of the growths are shown in Figure 2. Manufacturers 2, 3 and 4 had relatively low crack densities while manufacturers 1, 5, and 6 had very high crack densities. Seeds from different manufacturers cracked in different ways. For instance, some had clear crystallographic cracking following M-planes while others regularly had a circular component. Unfortunately since we don't have knowledge of each manufacturer's HVPE process, it is difficult to determine what HVPE growth conditions are required for suitable ammonothermal growth. However it is clear that HVPE seeds require several characteristics; low (002) and (201) FWHM values as measured by XRC, uniformity across the sample, and avoidance of techniques that lead to strong grain boundaries.

Focusing efforts on ammonothermal growth using seeds that generated thick crystals with a lower crack density, SixPoint Materials was able to further reduce cracking from two manufacturers to zero observable cracks by changing the growth conditions. However, we discovered that significant variability existed from seeds acquired from the same manufacturer, which resulted in very different growths as shown in Figure 3. We have since come up with a method to identify which seeds are suitable 
for thick crystal growth by the NEAT method. Using SixPoint Materials' NEAT method, we are now able to reproducibly obtain near crack free crystals in our small autoclaves on appropriate seeds. Due to the 1 " internal diameter size limitation in our small research autoclaves, we typically cut a 2" HVPE crystal into 4 strips. Regrowth on these strips are typically crack free or near crack free with excellent crystal quality as measured by a modified XRD grid as described above. Crystal quality as measured by XRD is routinely improved over the initial seed as shown in Figure 4. Some of this improvement in XRC values can be attributed to improved curvature. However even on samples with poor curvature we typically see a reduction in FWHM values. Part of this reduction is likely due to the imperfect surface finishing of the initial HVPE seed's nitrogen surface. However, dislocation counting does indicate that we routinely decrease our dislocation density from low to mid $10^{6} \mathrm{~cm}^{-2}$ to low to mid $10^{5} \mathrm{~cm}^{-2}$.

While we can report an extremely good radius of curvature of over $570 \mathrm{~m}$, these high curvature values are not stable with thickness, as the curvature always changes direction from the seed producing infinite curvature at a critical thickness. However we have improved final curvature of thick crystals with a stable curvature from a range of 13 meters to a general range of 3-20 meters in the opposite direction from the HVPE seed. This curvature flip is likely caused by the slight differences in lattice spacing due to the relatively high impurity content from ammonothermal growth as compared to HVPE seeds. The measurements used to calculate curvature are shown in Figure 5 for the same crystals as Figure 4. The radius of curvature is calculated from the slope of the line, making it easy to visualize a curvature flip as when the slope changes from negative to positive. Clear flipping of the curvature is observed. These new curvature values are uniform across the crystal and don't degrade with increased crystal thickness. In a similar plot on a crystal with cracks, a discontinuity or jump can be observed at each crack.

SixPoint Materials' focus in the small autoclaves currently is to produce crack free $10 \mathrm{~mm} \times 10 \mathrm{~mm}$ pieces that we can supply to research partners for device testing. Each initial seed results in a growth that can be sliced into a minimum of 3 wafers of the same size as the starting seed placed into the autoclave. These wafers are then diced into crack free $10 \mathrm{~mm}$ x $10 \mathrm{~mm}$ pieces as shown in Figure 6. SixPoint is now working with 
third party collaborators, including University of Notre Dame and Cornel University for device testing to ensure the samples are useful.

\section{Conclusion}

Ammonothermal growth has several advantages as far as scalability and ability to produce high quality free standing GaN crystals. By testing multiple manufacturer's free standing HVPE seeds, we were able to determine features of the seeds that are required for crack free regrowth. Out of the possible HVPE seed providers tested, only 2 or 3 appear to produce seeds with a compatible grain structure for thick regrowth by our NEAT method.

By improving the growth condition and carefully selecting seed crystals, SixPoint Materials is now capable of producing a small quantity of high quality $10 \mathrm{~mm} \times 10 \mathrm{~mm}$ crystals from a single seed. Those crystals are being provided to research institutes and companies for evaluation as substrates for devices.

The ability to turn one wafer into 3-5 high quality crack free wafers is an important metric to demonstrate the increased feasibility of bulk GaN crystal production by our NEAT method at SixPoint Materials.

Acknowledgement: Part of this work was conducted with support from the DOE ARPAE (Award No. DE-AR000045) 


\section{References}

1 A. Usui, H. Sunakawa, A. Sakai, A. Yamaguchi, Jpn. J. Appl. Phys. 36 (1997) L899.

2 R.J. Molnar, W. Gotz, L.T. Romano, N.M. Johnson, J. Cryst. Growth 178 (1997) 147.

3 C. A. Hurni, A. David, M. J. Cich, R. I. Aldaz, B. Ellis, K. Huang, A. Tyagi, R. A. DeLille, M. D. Craven, F. M. Steranka, and M. R. Krames, Appl. Phys. Lett. 106, 031101 (2015)

4 Briere, M. CS MANTECH Conference, May 17th-20th, 2010, Portland, Oregon, USA

5 Z. Hu, K. Nomoto, B. Song, M. Zhu, M. Qi, M. Pan, X. Gao, V. Protasenko, D. Jena, and H. Grace Xing, Appl. Phys. Lett. 107, 243501 (2015)

6 K. Fujito, S. Kubo, H. Nagaoka, T. Mochizuki, H. Namita, and S. Nagao, J. Cryst. Growth 311(2009) 3011

7 H. Yamane, M. Shimada, T. Sekiguchi and F.J. DiSalvo, J. Cryst. Growth 186 (1998) 8.

8 F. Kawamura, M. Morishita, K. Omae, M. Yoshimura, Y. Mori and T. Sasaki, Jpn. J. Appl. Phys. 42 (2003) L879.

9 Y. Mori, Y. Kitaoka, M. Imade, F. Kawamura, N. Miyoshi, M. Yoshimura and T. Sasaki, Phys. Status Solidi (a) 207 (2010) 1283

10 D. Ehrentraut, R. Pakalapati, D. Kamber, W. Jiang, D. Pocius, B. Downey, M. McLaurin, and M. D’Evelyn, Jpn. J. Appl. Phys. 52 (2013) 08JA01

11 R. Dwiliński, R. Doradziński, J. Garczyński, L. Sierzputowski, A. Puchalski, Y. Kanbara, K. Yagi, H. Minakuchi and H. Hayashi, J. Cryst. Growth 310 (2008) 3911.

12 T. Hashimoto, E. Letts, M. Ikari, Y. Nojima, J. Cryst. Growth 312 (2010) 2503.

13 S. Pimputkar, S. Kawabata, J.S. Speck, and S. Nakamura, J. Cryst. Growth 403 (2014) 7

14 T. Sochacki; M. Amilusik; B. Lucznik; M. Boćkowski; J. L. Weyher; G. Nowak; B. Sadovyi; G. Kamler; I. Grzegory; R. Kucharski; M. Zajac; R. Doradzinski and R. Dwilinski, SPIE Proceedings Vol. 8625, (4 March 2013) 
Figure Captions

Table 1 An overview of the different mean XRC FWHM measurements for the (002) and (201) reflections from the 6 different manufacturer's seeds using a small beam spot, $0.3 \mathrm{~mm} \times 0.3 \mathrm{~mm}$.

Figure 1 Plotted grid profiles of the nitrogen surface (002) and (201) XRC FWHM values of six different manufacturer's seeds.

Figure 2 Typical images observed of wafers obtained by ammonothermal growth on the different manufacturer's seeds. Considerable fluctuations in crack densities can be observed. Growths on seeds produced by manufacturers 2, 3 and 4 had much lower crack densities than 1,5 and 6. Graph paper marks $1 \mathrm{~mm}$ increments. Wafers were cut to approximately 550 microns in thickness.

Figure $3 \mathrm{GaN}$ wafers produced from crystals grown on seeds from the same manufacturer. Difference in crack density was primarily caused by differences in the underlying seed crystal.

Figure 4 XRC FWHM values for both the (002) and (201) reflection from both the original seed and grown crystals for 2 different growths. A clear improvement is observed after growth. Crystals were grown to $\sim 3 \mathrm{~mm}$ in thickness.

Figure 5 XRC angle value for the (002) reflection from the original seed and grown crystals from the same crystals as Figure 4. A clear curvature flip is consistently observed.

Figure 6 Typical crystal being produced in our small autoclaves. The crystal was sliced by a multi-wire saw and then diced into fifteen $10 \mathrm{~mm} \times 10 \mathrm{~mm}$ pieces. 
Table 1

Letts et al.

\begin{tabular}{|c|c|c|c|c|c|}
\hline & \multicolumn{2}{|c|}{$\begin{array}{c}\omega 002 \\
\text { FWHM } \\
\text { (arcsec) }\end{array}$} & \multicolumn{2}{|c|}{$\begin{array}{c}\omega 201 \\
\text { FWHM } \\
\text { (arcsec) }\end{array}$} & $\begin{array}{c}\text { Global } \\
\text { Curvature } \\
\text { (m) }\end{array}$ \\
\hline & Mean & $1 \sigma$ & Mean & $1 \sigma$ & Mean \\
\hline Manufacturer 1 & 582 & 16 & 534 & 173 & 3.27 \\
\hline Manufacturer 2 & 124 & 21 & 59 & 9 & -27.51 \\
\hline Manufacturer 3 & 99 & 15 & 94 & 17 & -1.53 \\
\hline Manufacturer 4 & 180 & 6 & 301 & 32 & -32.79 \\
\hline Manufacturer 5 & 118 & 55 & 156 & 85 & -5.21 \\
\hline Manufacturer 6 & 194 & 25 & 299 & 46 & 2.48 \\
\hline
\end{tabular}

Figure 1

Letts et al.

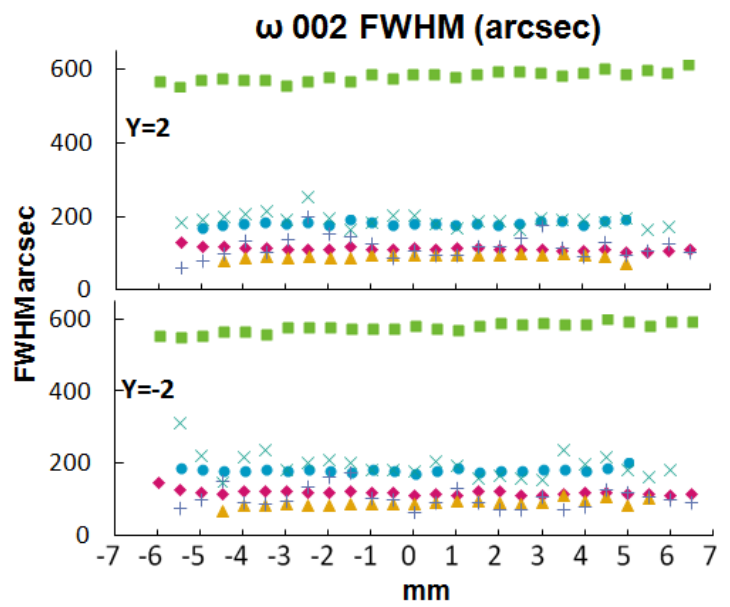

Manufacturer 1

Manufacturer 2
Manufacturer 3 A

Manufacturer 4

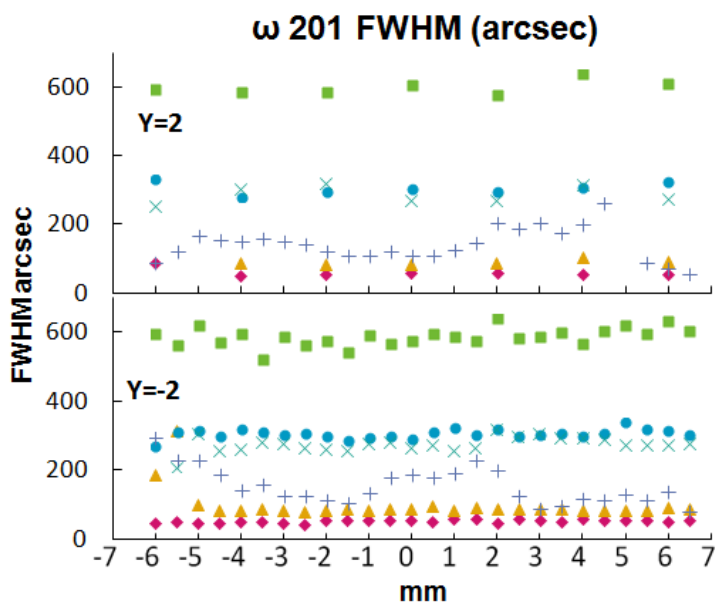

Manufacturer $5+$

Manufacturer $6 \times$ 
Figure 2

Letts et al.
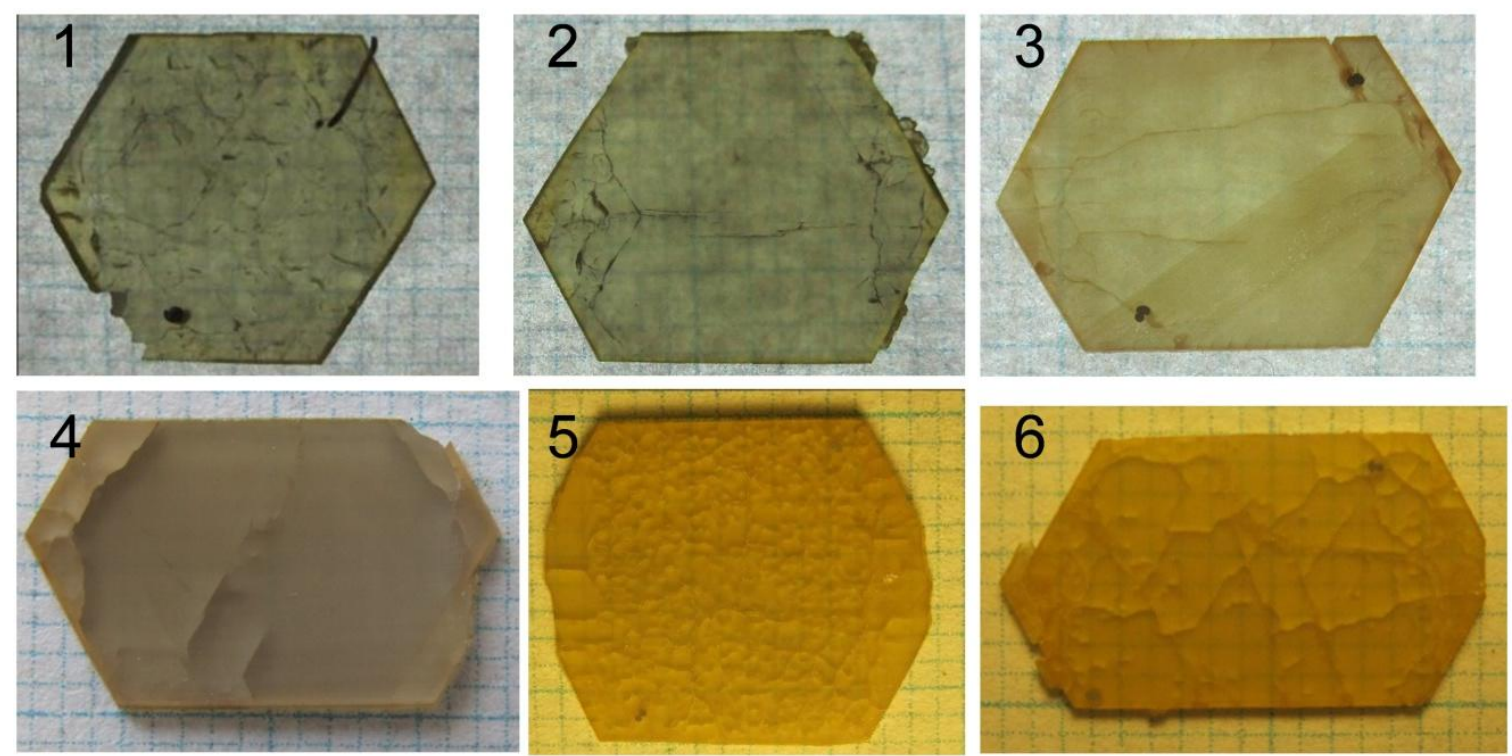

Figure 2 Grayscale

Letts et al.
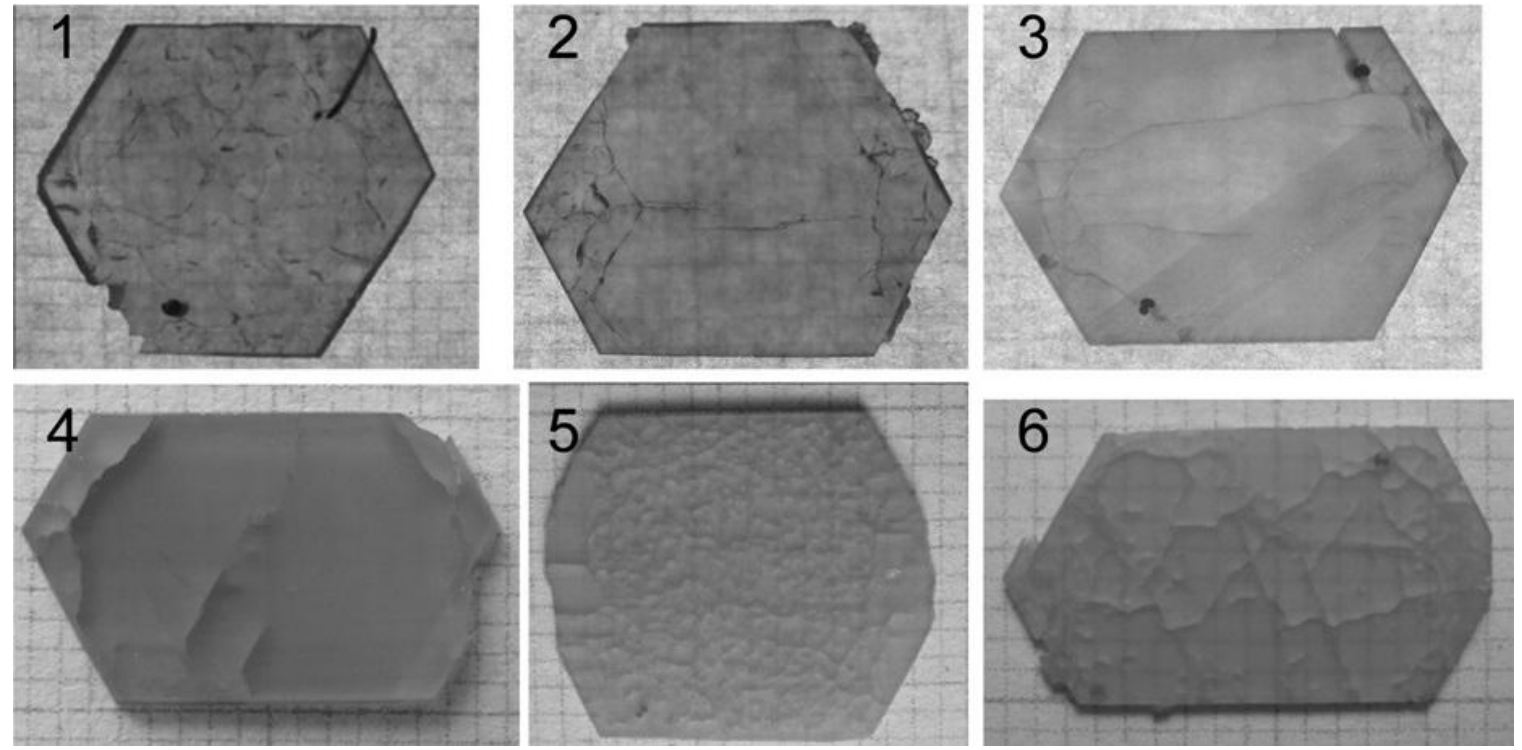
Figure 3

Letts et al.
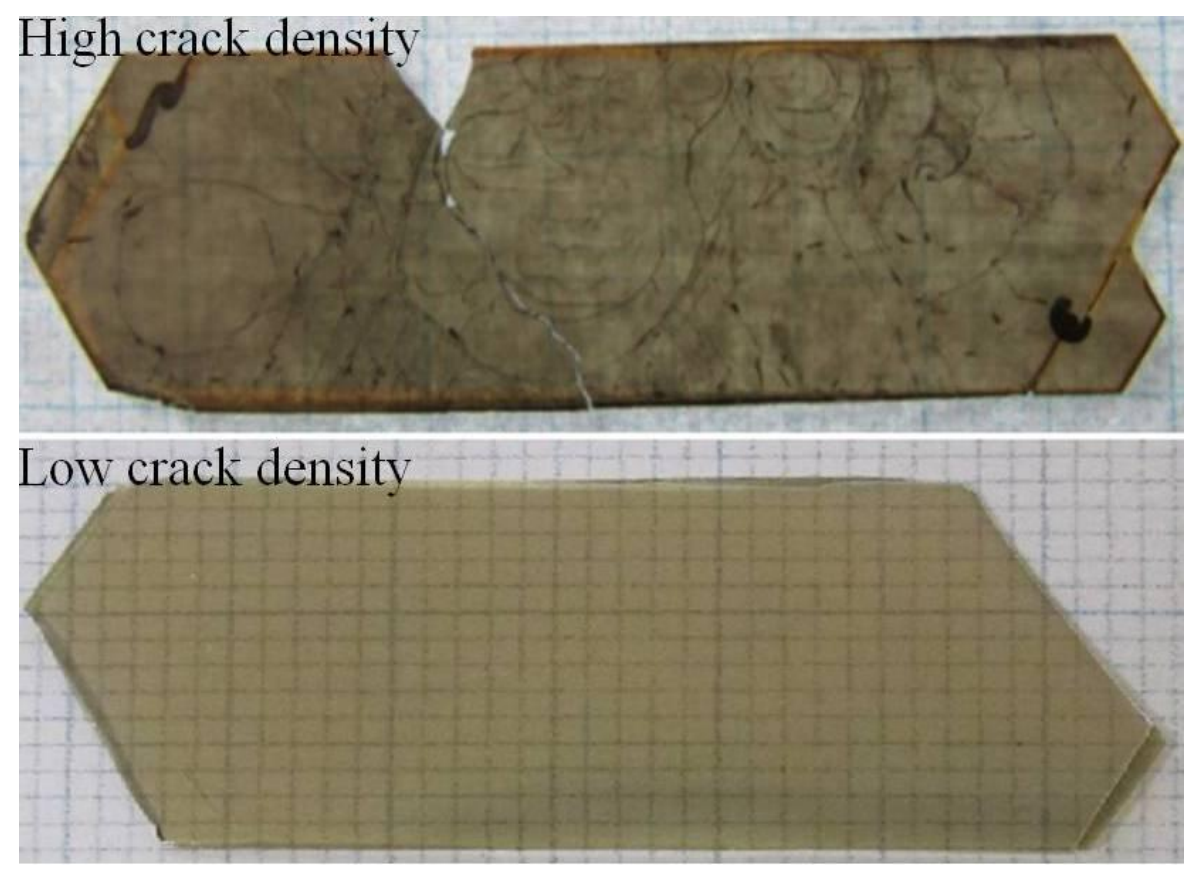

Figure 3 Grayscale

Letts et al.
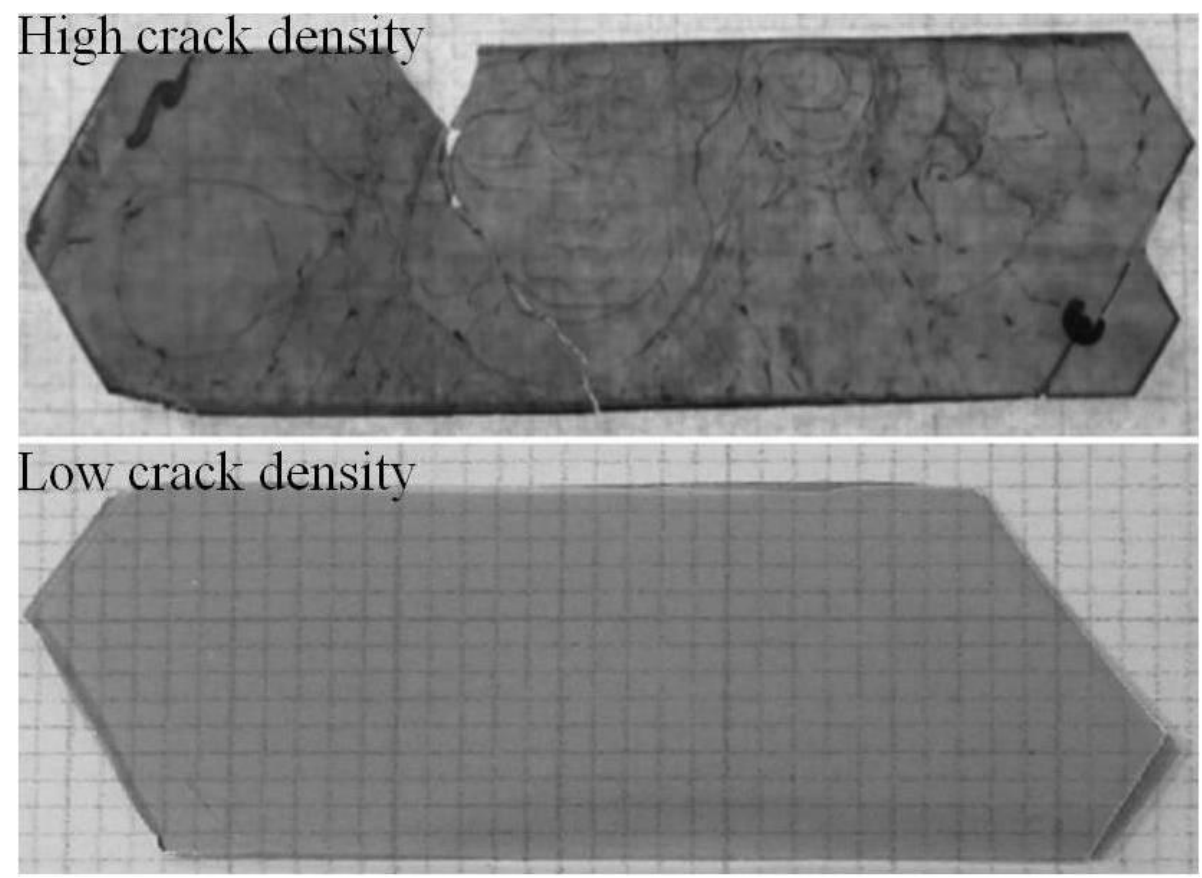
Figure 4

Letts et al.
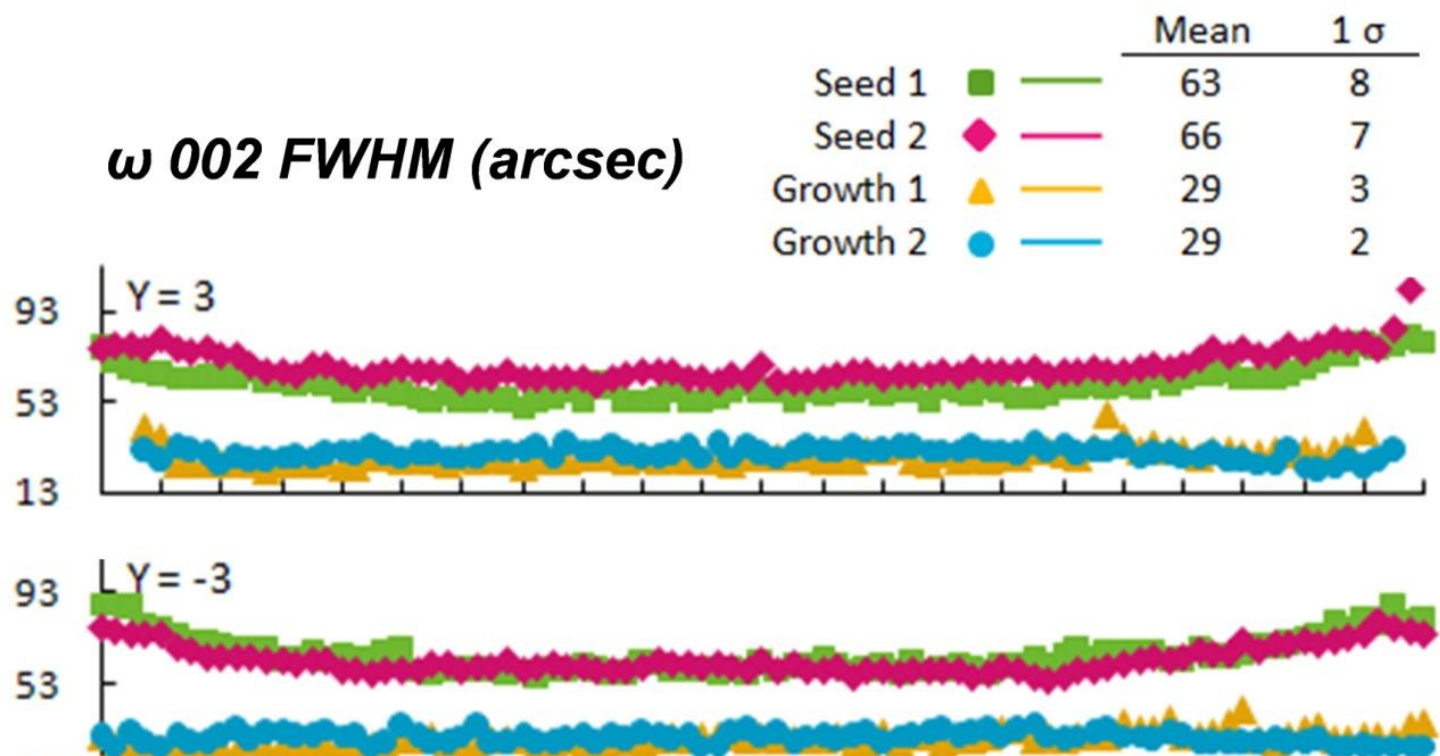

13

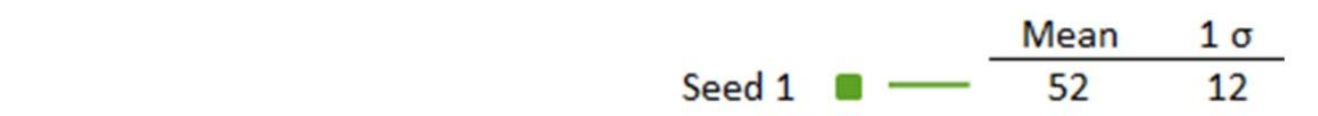

w 201 FWHM (arcsec) $\begin{aligned} \text { Seed 2 } & - \\ \text { Growth 1 } & 46\end{aligned}$

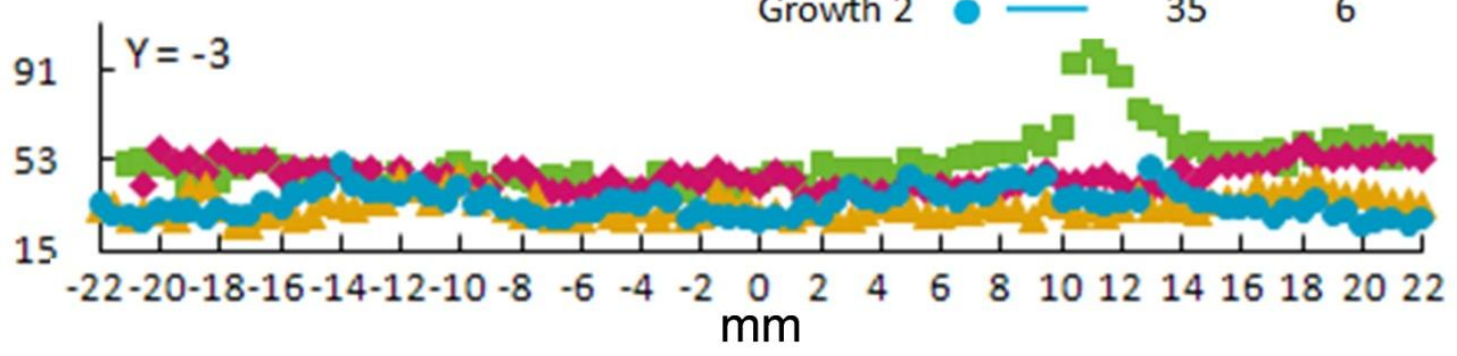


Figure 5

Letts et al.

\section{Global Curvature (m)}

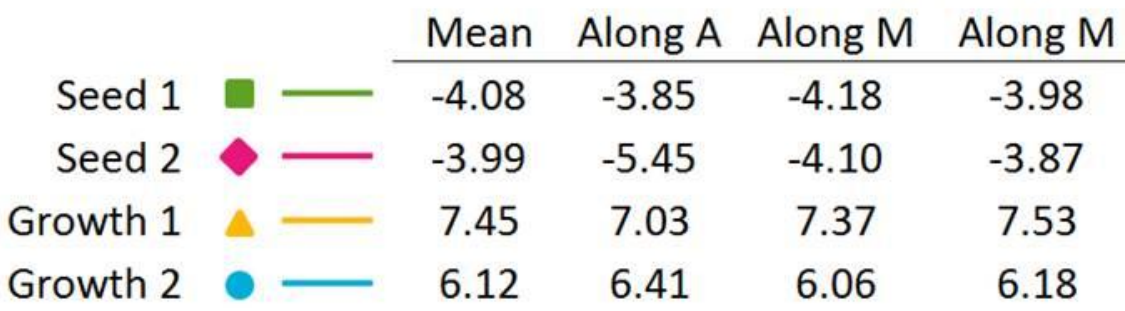

\section{Along A Direction}
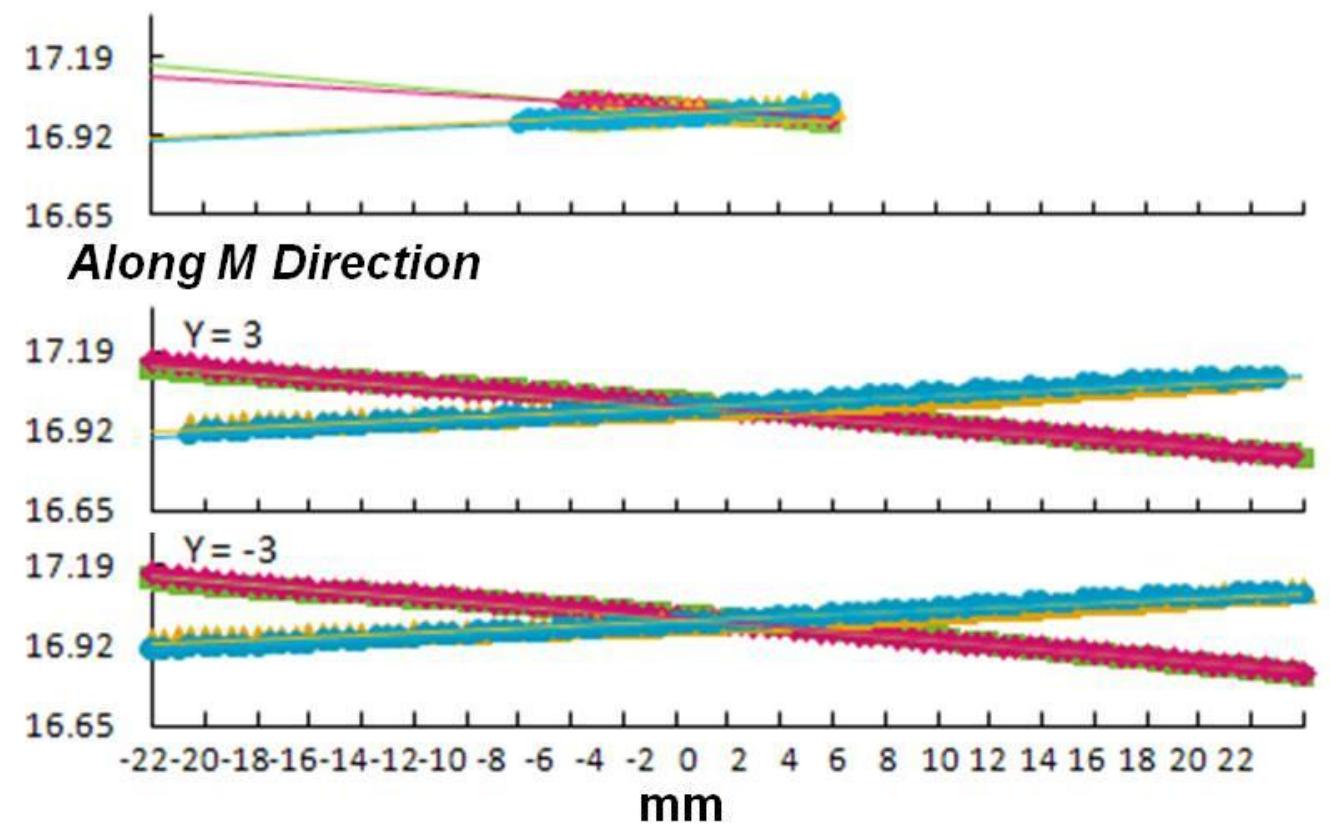
Figure 6

Letts et al.

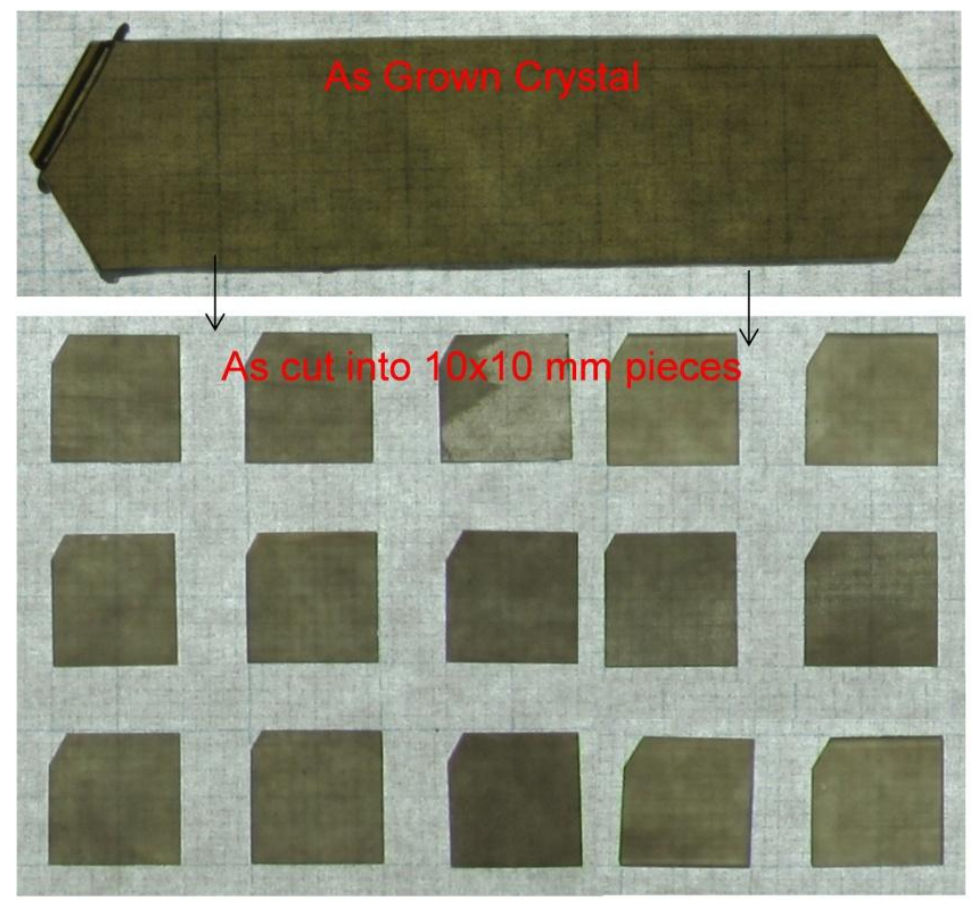

Figure 6 Grayscale

Letts et al.

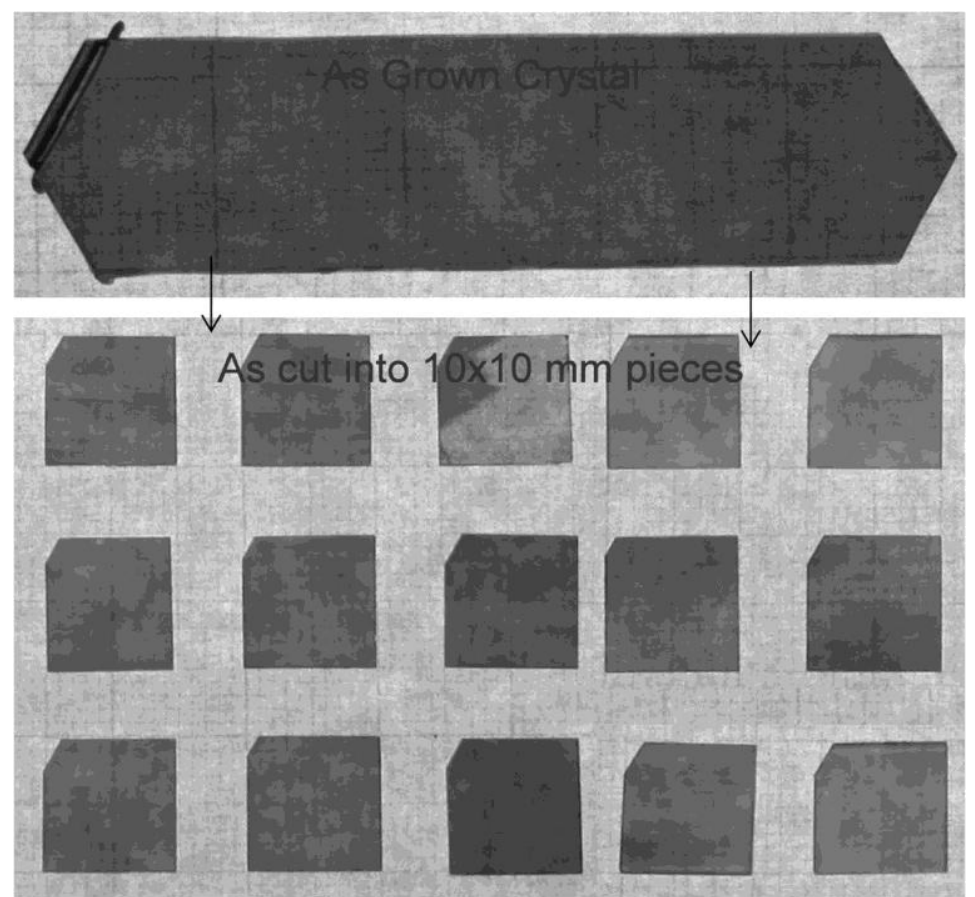

